\title{
Designing Systems for Health Promotion and Autonomy in Older Adults
}

\author{
Helena Lindgren ${ }^{1}$ and Ingeborg Nilsson ${ }^{2}$ \\ ${ }^{1}$ Department of Computing Science, Umeå University, SE-901 87 Umeå, Sweden \\ ${ }^{2}$ Department of Community Medicine and Rehabilitation, Unit for Occupational Therapy, \\ Umeå University, SE-901 87 Umeå, Sweden \\ helena@cs.umu.se, ingeborg.nilsson@umu.se
}

\begin{abstract}
The inclusion and autonomy of older people in the society where large parts of the life is organized with computer and Internet use as means is addressed in an ongoing project in the rehabilitation and health domains. Part from investigating the potentials of using ICT for rehabilitation of older people with limited or no computer skills, the aim for the project is to develop methods and tools for the purpose, and also for the interaction design domain where systems are developed for older people. The resulting methods are used for informing the design of the system in an iterative process.
\end{abstract}

Keywords: Interaction design, activity theory, health care, evaluation.

\section{Introduction}

Activities are, to an increasing extent, re-organized to involve the web as a means, as source of information and entertainment, channel for communication, base for social environments and for administration of individual's personal and social life. This trend excludes persons who have not access, knowledge or the skills to use computers and Internet facilities from a large part of the society and often also parts of the social life of the immediate family. To a large extent, older persons are characterized by this exclusion and would benefit from purposeful designed systems aimed at supporting the execution of activities of their choice. This category of aids to overcome hindrances to perform needed activities in daily life constitutes a significant asset for occupational therapy interventions. As part of the professional reasoning to evaluate activity barriers in individual clients, the adjustments of the environment in which the activity is to be performed is done on a regular basis in the rehabilitation process. Therefore, the professional assessment of the needs for certain computer and Internet based aids constitutes an activity that will increase in importance by the increased demands of ICT-mediated distributed activities in people's daily lives. However, this requires also the development of tools and methods for the rehabilitation domain for facilitating the integration of the task into daily practice.

The rehabilitation process is also a process distributed over time and location, sometimes involving additional actors such as family, where members often are located in other parts of the country. Computer and web based tools for investigation and follow-ups could easily overcome these factors, and may consequently 
enhance the client's role and influence over his or her rehabilitation process if used as a common tool for choices and adjustments of interventions. Therefore, a pilot study was initiated with the aims to investigate to what extent ICT can be used as a common tool in the rehabilitation process by both occupational therapist and an older patient. In this pilot study the possibilities are explored for such a tool in rehabilitation of activity impairments in older people and where the persons may not have any or very limited experience of computer use. Specifications and prototypes for such a tool are being developed. Of central concern in our work, is also the need for tools and guidelines for the process, both regarding the rehabilitation domain and for the domain of interaction design for older adults. Developing systems aimed to be used by older persons with limited experiences of computer use is a challenging task (e.g., [1, 2, 3, 4]). This process requires both a carefully organized design process methodologically, suitable for the inclusion of user representatives in the design process, and interaction designs that meet the special needs of older persons.

\section{Methods, Material and Procedure}

An activity-oriented participatory design process with heavy emphasis put on the activities in focus was applied, involving two occupational therapists, a computer scientist and a group of older volunteers. This was accomplished by using activity theory and the notion of ZPD (zone of proximal development) as the theoretical framework for data gathering, analysis and re-design $[5,6,7]$. The activities observed ranged from occupational therapists investigating the participants, the participants performing activities of their choice, and the dialogue between the two for the purpose of follow up on interventions. Interviews and questionnaires were used continuously. The occupational therapist's evaluation of activity status was used as starting point, in which activity hinders, personal resources, needs and interests were identified in eight older persons who volunteered to participate in the study. The persons comprised one man and seven women between the ages of 65-75, who did not have any or very limited experiences of using computers and none using the web.

A structured protocol emerged from the results of the initial assessment phase. The protocol were used for valuing and documenting the individual's development of skills and ability in Internet-mediated activity, identify reasons for breakdown situations, and to formulate questions for interviews and computer-based questionnaires. These were used after each session for assessing the subjective views and attitudes towards computer use. Furthermore, the protocol was used for informing the re-design of prototypes.

Based on the activity analyses and a literature review, initial prototypes of a virtual café environment were developed and integrated in an already existing physical Internet café environment for older people. The subjects participated in seven to eight organized occasions of 1,5 hours, during a period of two months. One woman did not proceed to participate in the café environment due to health reasons. 


\section{Results}

The major purpose of occupational intervention is to increase autonomy in performing activities in fields of interest and of importance for the individual. To summarize the results, all of the participants discovered new values that could be added to their activities by using computers and the web as tools. This includes new and easily accessed sources of information and knowledge, channels to organizations of interest where they are engaged, finding and communicating with old friends and family, developing interests. In addition, four persons expressed that it is simply fun to use the computer and that the enjoyment increases with control and skills. This is said in contrast to the remaining skeptical person who saw little use for, or enjoyment in computer-based activities, part from using the computer for keeping up with news.

Three levels of complexity in web pages were described by Dickinson and colleagues [4] where the first level could be seen as a "walled garden", hiding large parts of the web from the user with content fully controlled by the development team. The second layer allowed some external content that adhered to high standards of accessibility and navigation structures, while the third allowed any content of the web. A similar approach was taken in this study, increasing the complexity judged based on what lied in the next zone of development and based on what the individuals' wished to do during the next session. All participants wanted to do things eventually, which involve external web pages (some tried on their own in their homes but got lost among frames, different routines for logging in, etc.), therefore, handling such events were included in later sessions as part of their main activities. Maintaining a consistent basic structure was necessary in order to reduce the amount of new things to internalize. A resistance to change of structures was expressed, the participants wanted to be able to reuse knowledge as much as possible and not having to deal with several different ways of doing what they perceived as being the same thing. The participants found it hard to grasp differences between similar phenomenon, and one way to distinguish between them was by the procedure to use them (e.g., web addresses vs. searching by keywords, logging in). Therefore, one strategy to handle breakdowns was to start over from a point where they were familiar, which was a role the basic structure served. Therefore, this basic structure, which essential served as a home page, was adjusted to become more distinct from the rest of the web pages and easy to recognize among many open windows.

The iterative process of evaluation and re-design during the period of use, enabled the participants to contribute to the design of the system by trying to do what they wanted to be able to do, reflecting on their own performance and experienced computer-related phenomenon, without having to express explicit opinions about design choices. The latter seemed to be very difficult, since they did not distinguish differences between pages, objects, their properties, etc., to the extent that was necessary for having a specific opinion, and even less having the vocabulary to express an opinion in interviews or in questionnaires. What they did express though, was general desires for alternative ways of interacting with the computer. In spite of the simplified initial designs with introduction and support to practice basic skills, they had significant difficulties in particularly controlling the mouse and using controls in the user interface. An alternative approach to the first acquaintance to the use of computers is being developed using touch functionality. 


\section{Conclusions}

This paper reports on results from a pilot study aimed at developing prototypes for activity support involving older novice computer users in the process. A starting point for development is taken in the purposeful and desired activities to be supported, identified in eight individual cases. A protocol was developed based on activity theory and the notion of ZPD for informing the design of the system in an iterative process of re-design, use and evaluation. The seven older novice computer users who participated in the organized sessions increased their autonomy in computer-mediated activity to the extent that they are able to perform activities of their choice independently or with occasional support, and by partially using a structured web interface as starting point in case of breakdowns. By setting focus on the activity and using the protocol as base for assessing reasons for breakdown situations, the participants were able to contribute to the design process by reflecting on their performance of activities of their choice, without having to express explicit preferences among design choices or mastering computer-related phenomenon and terms. The method appears to be highly valuable in the context it has been used in this study, which is developing systems for the rehabilitation and health domain where the older novice actors need purposefully designed computer-based tools for increasing their autonomy and skills in daily activities. The protocol is being validated with additional users and activities in order to further investigate its usefulness for informing interaction design when involving potential users who may not be articulate about design choices. This is being done in the ongoing development of an extended system based on the results.

\section{References}

1. Dickinson, A., Arnott, J., Prior, S.: Methods for human - computer interaction research with older people. Behaviour \& Information Technology 26, 343-352 (2007)

2. Turner, P., Turner, S., van de Walle, G.: How older account for their experiences with interactive technology. Behaviour \& Information Technology 26, 287-296 (2007)

3. Hawthorn, D.: Interface design and engagement with older people. Behaviour \& Information Technology 26, 333-341 (2007)

4. Dickinson, A., Smith, M., Arnott, J., Newell, A., Hill, R.: Approaches to Web Search and navigation for older computer novices. In: Proceedings of the SIGCHI conference on Human factors in computing systems CHI 2007, pp. 281-290 (2007)

5. Vygotsky, L.: Mind in Society: The Development of Higher Psychological Processes. Harvard University Press, Cambridge (1978)

6. Engeström, Y.: Learning by Expanding: An Activity - Theoretical Approach to Developmental Research. Helsinki, Orienta-Konsultit (1987)

7. Bødker, S.: A human activity approach to user interfaces. Human-Computer Interaction 3 , 171-195 (1989) 